${ }^{5}$ R. Arnowitt, S. Deser, and C. W. Misner, in Gravitation, edited by L. Witten (Wiley, New York, 1962), Chap. 7.

${ }^{6} \mathrm{~A}$ similar formulation of Einstein's equations, though not as complete in its discussion, can be found in P. A. M. Dirac, Proc. Roy. Soc. (London) A246, 333 (1958).

${ }^{7}$ We follow the notation of Schutz, Ref. 2. In particular, Greek indices run from 0 to 3 , while Latin indices run from 1 to 3 , with these exceptions: $a$ and $b$ label the velocity potentials and run from 1 to 5 , while $m$ and $n$ label the Dirac $\varphi$ equations and run from 1 to 4 .

${ }^{8}$ Actually, Dirac's proof (Ref. 4 ) is for a system with finitely many degrees of freedom. We have generalized it to fields using the results of the Appendix.

${ }^{9}$ B. F. Schutz, Jr., in Proceedings of the Pittsburgh Conference on Relativity (Springer, Berlin, to be published).

${ }^{10}$ See Appendix B of Schutz, Ref. 2.

${ }^{11} \mathrm{~J}$. L. Synge, Relativity: The General Theory (NorthHolland, Amsterdam, 1966).

PHYSICAL REVIEW D

VOLUME 4, NUMBER 12

15 DECEMBER 1971

\title{
New Equation of Motion for Classical Charged Particles*
}

Tse Chin Mo and C. H. Papas

Electrical Engineering Department, California Institute of Technology, Pasadena, California 91109 (Received 15 March 1971)

\begin{abstract}
With the intuitive new ideas that (1) in classical electrodynamics, radiation reaction should be expressible by the external field and the charge's kinematics, (2) a charge experiences, in addition to the Lorentz forces, another "small" external force $e_{1} F^{\mu \lambda} \dot{u}_{\lambda}$ proportional to its acceleration, and (3) inertia plus radiation is balanced by these two external forces, we propose the new equation of motion,

$$
m \dot{u}^{\mu}-\left(2 e^{3} / 3 m\right) F_{\mathrm{ext}}^{\lambda \alpha} \dot{u}_{\lambda} u_{\alpha} u^{\mu}=e F_{\mathrm{ext}}^{\mu \lambda} u_{\lambda}+e_{1} F_{\mathrm{ext}}^{\mu \lambda} \dot{u}_{\lambda},
$$

where mass conservation requires $e_{1}=2 e^{3} / 3 m$. (The particle's spin is not considered in this work.) This equation for a classical charge is free from all the well-known difficulties of the Lorentz-Dirac equation. It conserves energy and momentum in a modified form in which the energy-momentum tensor contains a part $t^{\mu \nu}(x)$ made of a new field-charge interaction $\phi^{\mu}(x)$, in addition to the conventional "local" part made of $F_{\mathrm{ret}}^{\mu \nu}(x)$ and $\boldsymbol{F}_{\mathrm{e} x \mathrm{t}}^{\mu \nu}(x)$ only, and therefore it no longer satisfies the conventional "local" conservation laws. It predicts correct radiation damping, as demonstrated here by applying it to various cases of basic physical importance. Also, it implies that a massless particle follows a null geodesic and cannot interact with the electromagnetic field whether it be charged or not; this implication may add a new degree of freedom to the charge-conservation law.
\end{abstract}

\section{INTRODUCTION}

The equation of motion of a charged particle has been a subject of interest for many years. ${ }^{1}$ The equation now generally accepted was obtained by Dirac by decomposing the energy-momentum tensor of the retarded self-field into a sum that renormalizes mass and a difference that gives reaction. ${ }^{2}$ An explanation and rederivation based on an absorber mechanism was provided by Wheeler and Feynman. ${ }^{3}$ However, as is well recognized, the Lorentz-Dirac equation has certain inherent difficulties. First, it involves the derivative of the acceleration and hence needs one extra condition, in addition to the Newtonian initial conditions, to determine the motion. Second, it gives runaway solutions which can be avoided only by artificially presenting a preacceleration. ${ }^{4}$ Third, in certain cases it implies that the external energy supplied to the particle goes only into kinetic energy, and radiation is created from an "acceleration self energy" which becomes more and more negative and is unphysical. It is the purpose of this work to obtain a new equation that is free from these difficulties, agrees with existing laboratory results, and predicts new phenomena that can distinguish the new equation from the old one and test its validity.

\section{THE NEW EQUATION}

By following the old idea of expressing the radiation reaction of a charged particle only by its kinematical quantities, it is not possible to construct an equation that includes reaction in a form simpler and more satisfactory than the LorentzDirac one. However, in classical electrodynamics in an inertial frame $\mathrm{f}^{5}$ the only field that can accelerate a charged particle and make it radiate is the external electromagnetic field $F_{\text {ext }}^{\mu \nu}$. Accordingly, radiation reaction should be expressible by $F_{\text {ext }}^{\mu \nu}$ 


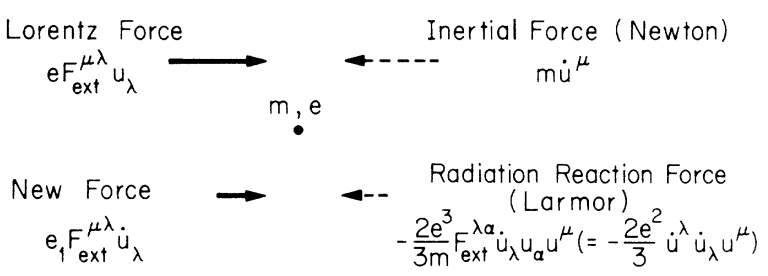

FIG. 1. The physical interpretation of the new equation.

and the particle kinematics. On the other hand, since a charge $e$ at rest experiences only an electric force $e \overrightarrow{\mathrm{E}}$ and in motion experiences an additional magnetic force $e \overrightarrow{\mathrm{v}} \times \overrightarrow{\mathrm{B}}$, which together make up $e F_{\text {ext }}^{\mu \lambda} u_{\lambda}$, it is natural to assume that when accelerating, a charge experiences still another aspect of the same external electromagnetic field -a new force $e_{1} F_{\text {ext }}^{\mu \lambda} \dot{u}_{\lambda}$ with $e_{1}$ a small constant (i.e., $e_{1} F_{\text {ext }}^{\mu \lambda} \dot{u}_{\lambda} \ll e F_{\text {ext }}^{\mu \lambda} u_{\lambda}$ in most physical cases) and $\dot{u}^{\lambda}$ $\equiv d u^{\lambda} / d s .^{6}$ We use geometrized units, $c=1, G=1$, $k=1$, with signature $\eta_{\mu \nu}=(1,-1,-1,-1)$, $\left\{x^{0}, x^{1}, x^{2}, x^{3}\right\} \equiv\{t, x, y, z\}, s$ the proper time and $u^{\mu}$ the four-velocity.

Now, given the motion $u^{\mu}(s)$ of a charge, the rate of radiated energy-momentum $\left(-2 e^{2} / 3\right) \dot{u}_{\lambda} \dot{u}^{\lambda} u^{\mu}$ is obtained by integrating Poynting's vector over the far-zone retarded sphere. ${ }^{7}$ By using the radiation-neglected equation $m \dot{u}^{\mu}=e F_{\text {ext }}^{\mu \lambda} u_{\lambda}$, this rate can be expressed as $\left(-2 e^{3} / 3 m\right) F_{\text {ext }}^{\lambda \alpha} \dot{u}_{\lambda} u_{\alpha} u^{\mu}$, which is roughly the expression for radiation in terms of $F_{\text {ext }}^{\mu \nu}$. Equating the inertia and radiation to the forces the charge sees through $F_{\text {ext }}^{\mu \nu}$, we have the new equation of motion (see Fig. 1)

$$
m \dot{u}^{\mu}-\left(2 e^{3} / 3 m\right) F_{\text {ext }}^{\lambda \alpha} \dot{u}_{\lambda} u_{\alpha} u^{\mu}=e F_{\text {ext }}^{\mu \lambda} u_{\lambda}+e_{1} F_{\text {ext }}^{\mu \lambda} \dot{u}_{\lambda},
$$

where the requirement that (1) be an identity after scalar multiplication by $u_{\mu}$ implies that $e_{1} \equiv 2 e^{3} / 3 m$ is indeed a small constant. For a system of charges, in (1) for the $i$ th charge $F_{\text {ext }(i)}^{\mu \nu}$ becomes $\sum_{j \neq i} F_{\text {ret }(j)}^{\mu \nu}$, where $F_{\text {ret }(j)}^{\mu \nu}$ is the retarded field of the $j$ th charge.

Also, just for reference, the MKS 3-vector form of (1) reads

$$
\begin{aligned}
m \frac{d}{d t} \frac{\overrightarrow{\mathrm{v}}}{\left(1-\beta^{2}\right)^{1 / 2}}+\frac{e^{2}}{6 \pi \epsilon_{0} c^{3}} \frac{\overrightarrow{\mathrm{v}}}{\left(1-\beta^{2}\right)^{3}}\left[\left(\frac{d}{d t} \vec{\beta}\right)^{2}-\left(\vec{\beta} \times \frac{d}{d t} \vec{\beta}\right)^{2}\right] \\
=e(\overrightarrow{\mathrm{E}}+\overrightarrow{\mathrm{v}} \times \overrightarrow{\mathrm{B}})+\frac{e^{3}}{6 \pi m \epsilon_{0} c^{3}}\left[\overrightarrow{\mathrm{E}} \frac{d}{d t} \frac{1}{\left(1-\beta^{2}\right)^{1 / 2}}+\left(\frac{d}{d t} \frac{\overrightarrow{\mathrm{v}}}{\left(1-\beta^{2}\right)^{1 / 2}}\right) \times \overrightarrow{\mathrm{B}}\right],
\end{aligned}
$$

where $\beta \equiv v / c$.

\section{GENERAL PROPERTIES OF THE NEW EQUATION}

(a) Mass conservation. Scalar multiplication by $u_{\mu}$ gives an identity and hence $m$ is constant.

(b) Self-evident radiation term. Scalar multiplication by $\dot{u}_{\mu}$ gives

$$
\left(-2 e^{3} / 3 m\right) F_{\mathrm{ext}}^{\lambda \alpha} \dot{u}_{\lambda} u_{\alpha} u^{\mu}=\left(-2 e^{2} / 3\right) \dot{u}_{\lambda} \dot{u}^{\lambda} u^{\mu},
$$

which always represents radiation. This justifies the second term on the left-hand side of (1) as radiation reaction with $u^{\mu}$ determined by (1).

(c) Newtonian motion. No more than the first derivative of velocity is involved and, accordingly, motion is determined by the initial velocity and position and by $F_{\text {ext }}^{\mu \nu}$.

(d) No runaway solutions (see below).

(e) No preacceleration.

(f) Additional "effective" external field. Taking the radiation term in (1) to the right-hand side and combining it with $e_{1} F^{\mu \lambda} \dot{u}_{\lambda}$, we can interpret the total acceleration-and-external-field-dependent force as obtained from an effective field, ${ }^{8}$

$$
f^{\mu \nu} \equiv\left(2 e^{2} / 3 m\right) \dot{u}_{\lambda}\left(F_{\mathrm{ext}}^{\mu \lambda} u^{\prime \prime}-F_{\mathrm{ext}}^{\nu \lambda} u^{\mu}\right),
$$

in addition to $F_{\text {ext }}^{\mu \nu}$, which the charge feels through the Lorentz force.

(g) Energy-momentum conservation. Since energy-momentum conservation and Maxwell's equations imply the equation of motion, (1) causes changes in at least one of them. For radiation to be of the Larmor form as in (b), the conventional Maxwell's equations must be retained. Thus the electromagnetic energy-momentum tensor $T_{\text {em }}$ should be modified as

$$
T_{\mathrm{em}}^{\mu \nu}=T_{\mathrm{tot}}^{\mu \nu}-\sum_{i} T_{\mathrm{ret}(i)}^{\mu \nu}+t^{\mu \nu},
$$

where $t^{\mu \nu}$ is defined to satisfy (see Appendix)

$$
t^{\mu \nu},{ }_{\nu}=\frac{2 e^{2}}{3 m} \sum_{(i)} \dot{u}_{\nu(i)}\left(F_{\mathrm{ext}}^{\mu \nu} u_{(i)}^{\lambda}-F_{\mathrm{ext}}^{\lambda \nu} u_{(i)}^{\mu}\right) J_{\lambda(i)} \equiv V^{\mu}
$$

and the symmetry requirement. Here $T_{\text {tot }}^{\mu \nu}$ and $T_{\mathrm{ret}(i)}^{\mu \nu}$ have just the familiar form of $F^{\mu \lambda} F_{\lambda}^{\nu}$ $-\frac{1}{4} g^{\mu \nu} F_{\alpha \beta} F^{\alpha \beta}$, but with $F_{\text {tot }}^{\mu \nu} \equiv F_{\text {incident wave }}^{\mu \nu}+\sum_{i} F_{\text {ret }(i)}^{\mu \nu}$ and $F_{\mathrm{ret}(i)}^{\mu \nu}$ inserted in them, respectively. The sig- 
nificance of (3) and (4) is that by integrating $T_{\mathrm{em}, \nu}^{\mu \nu}$ $+T_{\mathrm{mass}, \nu}^{\mu \nu}=0$ over the 3 -space of the $i$ th charge, then $^{9}$

$$
\int_{(i)}\left(T_{\mathrm{em}}^{\mu \nu}+T_{\mathrm{mass}}^{\mu \nu}\right){ }_{\nu} d^{3} x=0 \Rightarrow \mathrm{Eq} .(1),
$$

where Maxwell's equations and $T_{\text {mass }}^{\mu \nu} \equiv \mu u^{\mu} u^{\nu}$ with proper mass density $\mu$ have been used, and no mass renormalization was involved. ${ }^{10}$

\section{SPECIAL CASES AND COMPARISON WITH THE LORENTZ-DIRAC EQUATION}

Now we shall examine the implication of the new equation (1) for certain basic physical situations and compare the results with those $e^{11}$ of the Lorentz-Dirac equation

$$
m \dot{u}^{\mu}=e F_{\text {ext }}^{\mu \nu} u_{\lambda}+\frac{2}{3} e^{2}\left(\ddot{u}^{\mu}+\dot{u}_{\lambda} \dot{u}^{\lambda} u^{\mu}\right) .
$$

(a) No external field, $F_{\mathrm{ext}}^{\mu \nu}=0$. Equation (1) directly gives $u^{\mu}=$ const, but for (6) this solution has to be "physically" singled out from the infinity of runaway solutions.

(b) Constant uniform electric field, $\overrightarrow{\mathrm{E}}=\overrightarrow{\mathrm{e}}_{x} E$. The new equation (1) gives $u^{\mu}=(\cosh \eta, \sinh \eta, 0,0)$, where $\eta \equiv C_{1}+e E s / m$, for initial velocity $\overrightarrow{\mathrm{v}}=\overrightarrow{\mathrm{e}}_{x} \tanh C_{1}$; the Lorentz-Dirac equation (6) gives $u^{\mu}=(\cosh \xi, \sinh \xi, 0,0)$, where $\xi \equiv K_{1}+K_{2} e^{s / \tau}+e E s / m$ and $\tau \equiv 2 e^{2} / 3 m$, which with the "physical" requirement $\dot{u}^{\mu}=0$ when $E=0$ implies $K_{2} \equiv 0$. Thus (1) yields the same solution as (6), but (1) works all by itself. Also, in this case, from (1) the radiation $\left(-2 e^{2} / 3\right) \dot{u}_{\lambda} \dot{u}^{\lambda} u^{\mu}=\left(2 e^{4} E^{2} / 3 m^{2}\right) u^{\mu}$ is equal to the new external force $e_{1} F_{\text {ext }}^{\mu \lambda} \dot{u}_{\lambda}$; but from the LorentzDirac equation, (6), it is supplied by the negative "self-acceleration energy" term $\left(2 e^{2} / 3\right) \ddot{u}^{\mu}$ and the "puzzle" of radiating without radiation reaction,

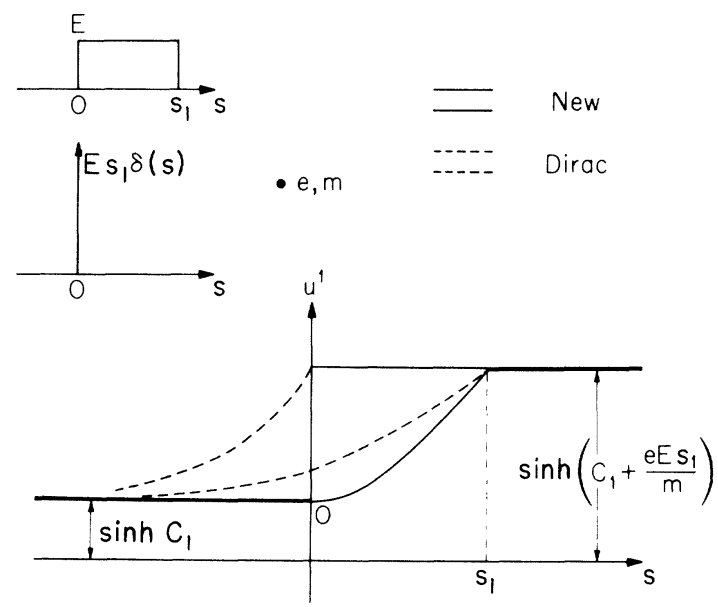

FIG. 2. Responses of the new equation and the LorentzDirac equation to rectangular and $\delta$ incident electric field. which really demonstrates another defect of Eq. (6), arises. ${ }^{12}$

(c) Incident rectangular pulse $\overrightarrow{\mathrm{E}}=\overrightarrow{\mathbf{e}}_{x} E$ for $0<s$ $<s_{1}$. Equation (1) gives

$$
u_{(N)}^{\mu}= \begin{cases}\left(\cosh C_{1}, \sinh C_{1}, 0,0\right), & s<0 \\ (\cosh \psi, \sinh \psi, 0,0), & 0<s<s_{1} \\ (\cosh \zeta, \sinh \zeta, 0,0), & s_{1}<s,\end{cases}
$$

where $\psi \equiv e E s / m+C_{1}$ and $\zeta \equiv e E s_{1} / m+C_{1}$. But (6), with the requirement $\dot{u}^{i}(\infty)=0$, gives

$$
u_{(\mathrm{LD})}^{\mu}= \begin{cases}(\cosh \alpha, \sinh \alpha, 0,0), & s<0 \\ (\cosh \sigma, \sinh \sigma, 0,0), & 0<s<s_{1} \\ (\cosh \zeta, \sinh \zeta, 0,0), & s_{1}<s,\end{cases}
$$

where

$$
\alpha \equiv C_{1}+(e E \tau / m)\left(1-e^{-s_{1} / \tau}\right) e^{s / \tau}
$$

and

$$
\sigma \equiv C_{1}+(e E \tau / m)\left(1-e^{\left(s-s_{1}\right) / \tau}\right)+e E s / m .
$$

Thus (8) represents preacceleration, whereas (7) shows that the electron does not respond until the pulse hits it. The limiting case of a $\delta$ pulse $^{2}$ is easily obtained by letting $s_{1} \rightarrow 0$ and keeping $E s_{1}$ constant. For this limit (7) gives simply a step jump in velocity which is due only to idealizing the incident wave as a $\delta$ function, whereas (8) gives a purely preaccelerational motion (see Fig. 2).

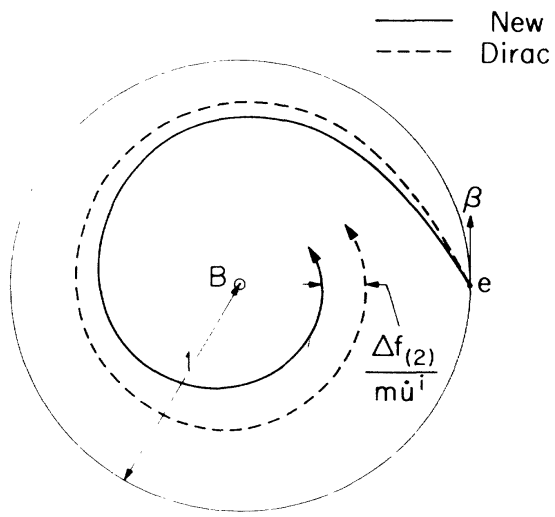

$$
\begin{aligned}
\frac{\Delta f_{(2)}}{m \dot{u}^{\prime}} & =\max \left[\frac{\beta^{2}}{\left(1-\beta^{2}\right)^{2}}\left(\frac{r_{c}}{r_{1}}\right)^{2}, \frac{\beta^{4}}{\left(1-\beta^{2}\right)^{3}}\left(\frac{r_{c}}{r_{1}}\right)^{2}\right] \\
& \sim 10^{-8} \text { for } e^{-} \text {at } \sim 5 \mathrm{BeV}, r_{1} \sim 1 \text { meter } \\
& \approx 1 \text { if } \frac{1}{1-\beta^{2}}\left(\frac{e^{2}}{m \epsilon_{0} C^{2}}\right)\left(\frac{m C}{e B}\right)^{-1} \gtrsim 1, \\
& \text { e.g. : } e^{-} \text {at } \sim 10^{n} \text { BeV, B } \sim 10^{b} \text { gauss, } b+2 n \gtrsim 10
\end{aligned}
$$

FIG. 3. Motions in perpendicular magnetic field and their deviations. 
(d) Motion perpendicular to uniform magnetic field $\overrightarrow{\mathrm{B}}=\overrightarrow{\mathrm{e}}_{\boldsymbol{x}} B$. In this case exact analytic solutions cannot be found for (1) and (6), but a perturbation method can be used to obtain and compare their total correctional forces which spiral the circular orbit inward (see Fig. 3) as a result of synchrotron radiation. ${ }^{13}$ Now the first-order corrections,

$$
\begin{aligned}
f_{(N 1)}^{\mu} \equiv \frac{2 e^{3}}{3 m} \dot{u}_{\lambda(1)}\left(F_{\mathrm{ext}}^{\mu \lambda}+F_{\mathrm{ext}}^{\lambda \alpha} u_{\alpha(1)} u_{(1)}^{\mu}\right) & =-\frac{2 e^{4} B^{2}}{3 m^{2}} \frac{\beta}{\left(1-\beta^{2}\right)^{1 / 2}}\left[\frac{\beta}{\left(1-\beta^{2}\right)^{1 / 2}} u_{(1)}^{\mu}+\left(0,0, \sin \frac{e B s}{m}, \cos \frac{e B s}{m}\right)\right] \\
& =\frac{2 e^{2}}{3}\left(\ddot{u}_{(1)}^{\mu}+\dot{u}_{\lambda(1)} \dot{u}_{(1)}^{\lambda} u_{(1)}^{\mu}\right) \equiv f_{(\mathrm{LD} 1)}^{\mu},
\end{aligned}
$$

are equal. Here $m \dot{u}_{(1)}^{\mu} \equiv e F_{\mathrm{ext}}^{\mu \lambda} u_{\lambda_{(1)}}$ and $u_{(1)}^{\mu}$ represent circular motion without radiation perturbation. The second-order corrections are

$$
\begin{aligned}
f_{(N 2)}^{\mu} \equiv & \frac{2 e^{3}}{3 m}\left[\dot{u}_{\lambda(2)}\left(F_{\mathrm{ext}}^{\mu \lambda}+F_{\mathrm{ext}}^{\lambda \alpha} u_{\alpha(1)} u_{(1)}^{\mu}\right)+\dot{u}_{\lambda(1)} F_{\mathrm{ext}}^{\lambda \alpha}\left(u_{\alpha(1)} u_{(2)}^{\mu}+u_{\alpha(2)} u_{(1)}^{\mu}\right)\right] \\
= & \frac{2 e^{3}}{3 m} F_{\mathrm{ext}}^{\lambda \alpha} \dot{u}_{\lambda(1)} u_{\alpha(1)} u_{(2)}^{\mu}+\frac{4}{9} \frac{e^{7} B^{3}}{m^{4}} \frac{\beta}{\left(1-\beta^{2}\right)^{3 / 2}} \frac{e B s}{m}\left[\frac{2 \beta}{\left(1-\beta^{2}\right)^{1 / 2}} u_{(1)}^{\mu}+\left(0,0, \sin \frac{e B s}{m}, \cos \frac{e B s}{m}+\frac{m}{e B s} \sin \frac{e B s}{m}\right)\right], \\
f_{(\mathrm{LD} 2)}^{\mu} \equiv \frac{2}{3} e^{2}\left(\ddot{u}_{(2)}^{\mu}+2 \dot{u}_{\lambda(1)} \dot{u}_{(2)}^{\lambda} u_{(1)}^{\mu}+\dot{u}_{\lambda(1)} \dot{u}_{(1)}^{\lambda} u_{(2)}^{\mu}\right) & \\
=\frac{2}{3} e^{2} \dot{u}_{\lambda(1)} \dot{u}_{(1)}^{\lambda} u_{(2)}^{\mu}+\frac{4}{9} \frac{e^{7} B^{3}}{m^{4}} \frac{\beta}{\left(1-\beta^{2}\right)^{3 / 2}} \frac{e B s}{m}\left[\frac{2 \beta}{\left(1-\beta^{2}\right)^{1 / 2}}\left(1+\frac{m}{2 e B s} \sin \frac{2 e B s}{m}\right) u_{(1)}^{\mu}\right. & \left.\quad+\left(0,0, \sin \frac{e B s}{m}-\frac{2 m}{e B s} \cos \frac{e B s}{m}, \cos \frac{e B s}{m}+\frac{m}{e B s} \sin \frac{e B s}{m}\right)\right] .
\end{aligned}
$$

Here the second-order total solution $u_{(2 t)}^{\mu} \equiv u_{(1)}^{\mu}+u_{(2)}^{\mu}$ which satisfies $m \dot{u}_{(2 t)}^{\mu}=e F_{\mathrm{ext}}^{\mu \lambda} u_{\lambda(2 t)}+f_{(N 1)}^{\mu}$ is the same for (1) and (6). Comparing the difference of second-order forces $\Delta f_{(2)}^{i} \equiv f_{(\mathrm{LD} 2)}^{i}-f_{(N 2)}^{i}$ and the first-order correction force $f_{(1)}^{i}$ to the main force $m \dot{u}^{i}$, we get

$\begin{array}{ccccc}\Delta f_{(2)}^{i} & f_{(1)}^{i} & m \dot{u}_{(1)}^{i} & \\ \frac{\beta^{2}}{\left(1-\beta^{2}\right)^{2}}\left(\frac{r_{c}}{r_{1}}\right)^{2} & \frac{\beta}{\left(1-\beta^{2}\right)^{1 / 2}}\left(\frac{r_{c}}{r_{1}}\right) & 1 & \text { for } & \frac{\beta^{2}}{1-\beta^{2}} \lesssim 1 \\ \frac{\beta^{4}}{\left(1-\beta^{2}\right)^{3}}\left(\frac{r_{c}}{r_{1}}\right)^{2} & \frac{\beta^{3}}{\left(1-\beta^{2}\right)^{3 / 2}}\left(\frac{r_{c}}{r_{1}}\right) & 1 & \text { for } & \frac{\beta^{2}}{1-\beta^{2}} z 1 .\end{array}$

Here $r_{1}$ is the radius of the circular orbit for $u_{(1)}^{\mu}$ and $r_{c}$ is the classical radius of the charged particle. Thus the new equation (1) predicts a faster inward spiraling than does Eq. (6) by the deviation $\Delta f_{(2)}^{i} / m \dot{u}_{(1)}^{i}$ compared to the main unperturbed orbit (Fig. 3). For a typical electron synchrotron of $5 \mathrm{BeV}, r_{1} \sim 5 \mathrm{~m}, r_{c}=2.8 \times 10^{-15} \mathrm{~m}$, this deviation is $10^{-8}$ - far below the quantum fluctuation of synchrotron photon emission. ${ }^{14}$ However, for highly energetic charged particles in a very strong electromagnetic field, as in astrophysical applications, ${ }^{15}$ where $\left(1-\beta^{2}\right)^{-1}\left(e^{2} / m \epsilon_{0} c^{2}\right)(m c / e B)^{-1} z 1$ (e.g., $b+2 n$ $z 10$ for electrons of energy $10^{n} \mathrm{BeV}$ in a $B=10^{b}$ $\mathrm{G}$ field) the deviation is large. In such strong fields the new equation (1) predicts orbits quite different than Eq. (6).

(e) Motion in Coulomb field $\overrightarrow{\mathrm{E}}=\left(q / r^{2}\right) \overrightarrow{\mathrm{e}}_{r}$. By a perturbation method as above, the first-order corrections are

$$
\begin{aligned}
f_{(N 1)}^{\mu}= & \frac{2 m^{2}}{3 q^{2}} \frac{\beta^{8}}{\left(1-\beta^{2}\right)^{5 / 2}}\left((1,0,0,0)-\frac{1}{\left(1-\beta^{2}\right)^{1 / 2}} u_{(1)}^{\mu}\right), \\
f_{(\mathrm{LD} 1)}^{\mu}= & \frac{2 m^{2}}{3 q^{2}} \frac{\beta^{7}}{\left(1-\beta^{2}\right)^{5 / 2}} \\
& \times\left((0,-\sin \Omega s, \cos \Omega s, 0)-\frac{\beta}{\left(1-\beta^{2}\right)^{1 / 2}} u_{(1)}^{\mu}\right),
\end{aligned}
$$

where $\Omega \equiv-m \beta^{3} / e q\left(1-\beta^{2}\right)$. It follows that $f_{(\mathrm{LD} 1)}^{i}$ has more backward tangential correction than $f_{\left(N_{1}\right)}^{i}$ and thus the Lorentz-Dirac orbit collapses faster than 
the new orbit by (Fig. 4)

$$
\frac{\Delta f_{(1)}^{i}}{m \dot{u}_{(1)}^{i}} \equiv \frac{\left(2 e^{3} / 3 m\right) \dot{F}_{\mathrm{ext}}^{i \lambda} u_{\lambda(1)}}{m \dot{u}_{(1)}^{i}} \sim \frac{e}{q} \frac{\beta^{3}}{\left(1-\beta^{2}\right)} .
$$

There are no experimental data on this deviation.

(f) Oscillating electric field, $\overrightarrow{\mathbf{E}}=\overrightarrow{\mathbf{e}}_{x} \cos \omega t$. (See

Ref. 16.) For initial velocity zero, the new equation (1) gives exactly

$u_{(N)}^{\mu}=\left(\left\{1+[(e E / m \omega) \sin \omega t]^{2}\right\}^{1 / 2},(e E / m \omega) \sin \omega t, 0,0\right)$,

which shows no damping because of the continuous supply of energy from the oscillating fields. Also the motion (14) is the same as that obtained from the radiation-neglected Newton's equation $m \dot{u}^{\mu}$ $=e F_{\text {ext }}^{\mu \lambda} u_{\lambda}$ because in this special case the radiation $\left(2 e^{4} E^{2} / 3 m^{2}\right) \cos ^{2} \omega t u^{\mu}$ is again equal to the additional external power-force $e_{1} F_{\text {ext }}^{\mu \lambda} \dot{u}_{\lambda}$. This result agrees with the usual Thomson scattering ${ }^{17}$ and says the latter is exact up to the order of neglecting the magnetic force from the incident wave. For Eq. (6), a perturbation force

$$
f_{(\mathrm{LD} 1)}^{\mu}=-\frac{2 e^{3} E}{3 m} \sin \omega t u_{(N)}^{0}\left(u_{(N)}^{1}, u_{(N)}^{0}, 0,0\right)
$$

shifts the oscillation phase forward and decreases the amplitude ${ }^{18}$ which deviates the motion from $u_{(N)}^{\mu}$ when $\omega \tau \gtrsim 1\left(\tau \sim 10^{-24} \sec\right.$ for $\left.e^{-}\right)$. But this cannot be checked by classical experimental methods because such highly energetic Compton scattering must be treated quantum electrodynamically.

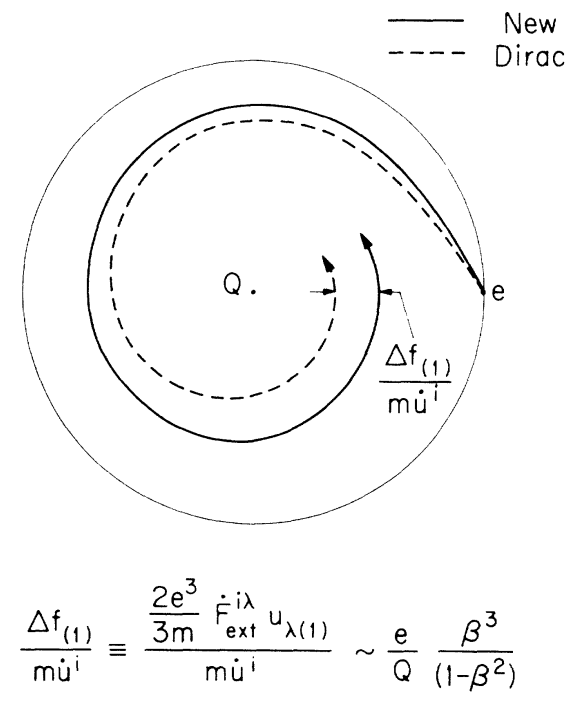

FIG. 4. Motions in a Coulomb field and their deviations.

\section{CONCLUSION}

The fact that (1) overcomes all former difficulties and predicts results not experimentally distinguishable from the Lorentz-Dirac equation in all laboratory cases of basic importance and the intuitive simplicity and soundness of the new ideas on which it is based lead us to suggest that the new equation (1) correctly accounts for radiation reaction in the motion of classical charged particles and should replace the celebrated Lorentz-Dirac equation of motion (6).

The new equation can manifest itself by predicting different motions and thus different radiation rates and patterns for highly energetic charges in very strong electromagnetic fields, e.g., as in astrophysical cases for electrons with $10^{n} \mathrm{BeV}$ in $10^{b}-\mathrm{G}$ fields such that $b+2 n z 10$. At present it does not seem trivial to find an action integral for (1). However, there is no valid action integral ${ }^{19}$ that leads to (6).

Also it can be shown that for $m=0$, Eq. (1) gives $\dot{u}^{\mu}=0$ and $u_{\lambda} u^{\lambda} \equiv 0$ independent of $F_{\text {ext }}^{\mu \nu}$. Thus a massless particle follows a null geodesic and cannot interact with the electromagnetic field whether it be charged or not. This might add a new degree of freedom to the charge-conservation law. The additional force appearing in (1) alters the conventional interaction $-J_{\mu} A_{\text {ext }}^{\mu}$. Thus this work is a first step of a new way toward including radiation reaction in curved space-time ${ }^{20}$ and may possibly lead to changes in quantum theory.

\section{ACKNOWLEDGMENTS}

The authors wish to thank Professor R. P. Feynman for enlightening discussions during this work. Also we thank Professor R. V. Langmuir, Professor J. Mathews, and Professor R. L. Walker for their comments; and Professor J. K. Knowles and Professor E. Sternberg for the proof included in the Appendix. Support from the U. S. Air Force Office of Scientific Research under Grant No. A FOSR-70-1935 is gratefully acknowledged.

\section{APPENDIX}

We investigate here the possibility of defining a $t^{\mu \nu}$ by (4), the construction of such a $t^{\mu \nu}$, and the indeterminacy of it. Now, if

$$
t^{\mu \nu}{ }_{, \nu}=V^{\mu}(x),
$$

where $V^{\mu}(x)$ is an arbitrary vector field, then a nontrivial symmetric tensor field solution $t^{\mu \nu}$ always exists (of course not unique) without any integrability condition, and its particular integral is

$$
t_{(p)}^{\mu \nu}=2 \phi^{(\mu, \nu)}-\eta^{\mu \nu} \phi^{\lambda}, \lambda,
$$


where the parenthesis means symmetrization and $\phi^{\mu}$ satisfies the familiar equation

$$
\square \phi^{\mu} \equiv \phi^{\mu, \lambda}, \lambda=V^{\mu} \text {. }
$$

The radiation and retardation conditions are included in the $\phi^{\mu}$. Equation (A1) in three dimensions is exactly the well-known stress equilibrium equation in continuum mechanics.

Thus, the general solution of (A1) is

$$
t^{\mu \nu}=t_{(p)}^{\mu \nu}+\Delta^{\mu \nu},
$$

where $\Delta^{\mu \nu}$ is any symmetric tensor satisfying $\Delta^{\mu \nu}$, $=0$. One way of choosing such $\Delta^{\mu \nu}$ harmonics is by using (A2) with a $\phi^{\lambda}$ that satisfies (A3) with $V^{\mu}=0$. Also the six degrees of indeterminacy of $t^{\mu \nu}$ are accounted for by these indeterminacies in $\Delta^{\mu \nu}$.

For the purpose of energy-momentum conservation (5), it is sufficient to let $\Delta^{\mu \nu}=0$ and choose $t^{\mu \nu}$ as the particular integral of (4), i.e.,

$$
t^{\mu \nu}=2 \phi^{(\mu, \nu)}-\eta^{\mu \nu} \phi_{, \lambda}^{\lambda},
$$

where

$$
\phi^{\mu}(x)=\frac{1}{4 \pi} \int d^{4} x^{\prime} \frac{\delta\left(t^{\prime}-t+\left|\overrightarrow{\mathbf{x}}-\overrightarrow{\mathbf{x}}^{\prime}\right|\right)}{\left|\overrightarrow{\mathbf{x}}-\overrightarrow{\mathbf{x}}^{\prime}\right|} V^{\mu}\left(x^{\prime}\right)
$$

with $V^{\mu}(x)$ defined in (4). Thus the additional energy-momentum change $t^{\mu \nu}$ in (3), which is defined by (4) and enters energy-momentum conservation as (5), indeed exists and is explicitly expressed by the field and currents as (A5) and (A6). Of course, the modified conservation law (5) includes this new $t^{\mu \nu}(x)$, thus differs from the conventional conservation law which involves only $F_{\text {ext }}^{\mu \nu}(x)$ and $F_{\text {ret }}^{\mu \nu}(x)$, and therefore it no longer satisfies the conventional "local" conservation laws. But the modified new conservation law is still a local one in the sense that it is a pointwise differential relation.
*Based partly on Caltech Antenna Laboratory Report No. 53, 1970 (unpublished), and on the presentation by T. C. Mo at the 1971 American Physical Society Meeting in New York [Bull. Am. Phys. Soc. 16, 118 (1971)] and the presentation by C. H. Papas before Academy of Sciences, Yerevan, U.S.S.R., 20 September 1970 [Izvestiia Akad. Nauk Arm. SSR, Fiz. $\underline{5}, 402$ (1970)].

${ }^{1} \mathrm{H}$. Lorentz, Theory of Electrons (Dover, New York, 1915), 2nd ed.; G. Schott, Electromagnetic Radiation (Cambridge U. P., Cambridge, England, 1912); L. Landau and E. Lifshitz, Classical Theory of Fields (AddisonWesley, Reading, Mass., 1962), 2nd ed., Secs. 75 and 76; F. Rohrlich, Classical Charged Particles (AddisonWesley, Reading, Mass., 1965); V. L. Ginzberg, Usp. Fiz. Nauk 98, 569 (1969) [Soviet Phys. Usp. 12, 565 (1970)]; S. A. Lebedeff, Phys. Rev. D 1, 1583 (1970).

${ }^{2}$ P. A. M. Dirac, Proc. Roy. Soc. (London) A167, 148 (1938).

${ }^{3}$ J. A. Wheeler and R. P. Feynman, Rev. Mod. Phys. 17,157 (1945).

${ }^{4}$ See any text treating classical radiation for the preacceleration-included integral-equation form of the Lorentz-Dirac equation.

${ }^{5}$ For noninertial frames, see T. C. Mo, J. Math. Phys. 11,2589 (1970).

${ }^{6}$ Here, of course, the existence of this new force $e_{1} F_{\mathrm{ext}}^{\mu \lambda} \dot{u}_{\lambda}$ is an ad hoc postulation.

${ }^{7}$ A. Schild, J. Math. Anal. Appl. 1, 127 (1960).

${ }^{8}$ If preferred, one can express $f^{\overline{\mu \nu}}$ by field quantities alone as $f^{\mu \nu} \equiv(e / m)\left(F_{\operatorname{ext} \lambda}^{\mu} I^{\lambda \nu}-F_{\text {ext } \lambda}^{\nu} I^{\lambda \mu}\right)$, where $I^{\mu \lambda}$ $\equiv \frac{2}{3} e\left(\dot{u}^{\mu} u^{\lambda}-\dot{u}^{\lambda} u^{\mu}\right)$ or $\dot{I}^{\mu \lambda} \equiv \frac{1}{2} F_{(-)}^{\mu \lambda}$ at the charge; and interpret $f^{\mu \nu}$ as the interaction of the external field with the self-difference radiation field.

${ }^{9}$ The mathematical detail is just similar to the familiar one; see, e.g., V. Fock, The Theory of Space, Time and Gravitation (Pergamon, New York, 1964), 2nd ed., Sec.
63; and J. L. Synge, General Relativity (Interscience, New York, 1960), p. 359.

${ }^{10} \mathrm{This}$ conservation can be equivalently stated as Eq.

(1) and Maxwell's equations $\Rightarrow\left(T_{\mathrm{em}}^{\mu \nu}+T_{\text {mass }}^{\mu \nu}\right),{ }_{\nu}=0$.

${ }^{11}$ G. N. Plass, Rev. Mod. Phys. 33 , 37 (1961); C. S.

Shen, Phys. Rev。 Letters 24, 410 (1970).

${ }^{12}$ For an attempted explanation of this "puzzle," see, e.g., T. Fulton and F. Rohrlich, Ann. Phys. (N.Y.) 9 , 499 (1960) ; D. Leiter, Intern. J. Theor. Phys. 3 , 387 (1970).

${ }^{13} \mathrm{~J}$. Schwinger, Phys. Rev. 75, 1912 (1949) ; D. H.

Tomboulian and P. L. Hartman, ibid. 102, 1423 (1956).

${ }^{14}$ M. Sands, Phys. Rev. 97, 470 (1955); T. Erber, Rev. Mod. Phys. 38,626 (1966).

${ }^{15} \mathrm{H}$. Y. Chiu and V. Canuto, Astrophys. J. 153, 157(L) (1968); Phys. Rev. Letters 22, 415 (1969); J. E. Gunn and J. P. Ostriker, ibid. 22, 728 (1969); F. Occhionero and M. Demianski, ibid. $\underline{23}, 1128$ (1969).

${ }^{16}$ Suggested by R. P. Feynman and also by P. A. M. Dirac in a private communication for a basic test in the case of a time-dependent field.

${ }^{17}$ L. Landau and E. Lifshitz, Ref. 1, Sec. 78.

${ }^{18}$ G. N. Plass, Ref. 11, Eqs. (92)-(95).

${ }^{19}$ The procedure of adding $A_{(-)}^{\mu} J_{\mu}$, where $A_{(-)}^{\mu}$ $\equiv \frac{1}{2}\left(A_{\mathrm{ret}}^{\mu}-A_{\mathrm{adv}}^{\mu}\right)$ and $J^{\mu} \equiv \rho_{(0)} u^{\mu}$, in the Lagrangian integrand and varying $u^{\mu}$ with $A_{(-)}^{\mu}$ fixed, then finally evaluating $F_{(-)}^{\mu \nu}$ at the charge to obtain the Lorentz-Dirac equations (6), is not a correct variation principle - see, for example, F. Rohrlich [Phys. Rev. Letters $\underline{12}, 375$ (1964)], since $A_{(-)}^{\mu}$ is a function of $u^{\mu}$.

${ }^{20}$ Simply postulating $d / d s$ of the new equation to be replaced by the invariant derivative $D / D S$ in a curved space-time may exclude possible couplings of the Riemann tensor $R^{\mu \nu \alpha \beta}$ into the motion. Also for a work following Dirac's idea, see B. S. DeWitt and R. W. Brehme, Ann. Phys. (N.Y.) 9, 220 (1960). 